\title{
LES TROIS STADES LARVAIRES DE COBBOLDIA LOXODONTIS
}

\author{
Par L. GEDOELST
}

Les larves de Cobboldia de l'éléphant d'Afrique ont été décrites dès 1897 par Brauer qui en avait examiné le stade tertiaire ; l'étude de ce même stade a été reprise plus récemment par Roubaud (1914) et par Rodhain et Bequaert (1919). En 1916, nous avons signalé sommairement une larve secondaire, mais la larve primaire n'a pas été observée jusqu'ici. Récemment, M. le $\mathrm{D}^{r}$ Larrousse nous a communiqué trois préparations microscopiques renfermant quatre larves primaires de Cobboldia loxodontis trouvées, avec de nombreuses larves tertiaires, dans l'estomac d'un rhinocéros autopsié par M. le prof. Brumpt en 1902, sur la rivière Ousnée, près de l'Omo. M. le $\mathrm{D}^{\mathrm{r}}$ Larrousse a bien voulu nous abandonner l'étude de ce matériel intéressant; nous tenons à lui adresser ici nos bien sincères remerciements pour sa gracieuse obligeance. Nous avons profité de cette occasion pour soumettre à une nouvelle étude la larve secondaire et les larves tertiaires que nous possédons et consignons ci-après les observations que nous avons faites sur les trois stades de la larve de Cobboldia loxodontis.

Larve primaire. - Des quatre exemplaires que nous avons examinés, trois étaient sur le point de muer et montraient à l'intérieur du tégument primaire l'appareil bucco-pharyngien et les stigmates antérieurs et postérieurs de la larve secondaire. Les dimensions de ces larves étaient de $6 \mathrm{~mm}$. à $6 \mathrm{~mm}$., 7 de longueur ; nous n'avons pas pris la largeur, celle-ci étant modifiée par l'écrasement qu'elles avaient subi sous le couvre-objet.

Le corps compte 12 segments bien distincts (fig. 1), dont le diamètre augmente notablement d'avant en arrière jusqu'au $5^{\circ}$ anneau et insensiblement de celui-ci au $10^{\circ}$; le $11^{\circ}$ est égal au précédent et le $12^{\circ}$ est légèrement réduit. La longueur des segments croît faiblement du $3^{\circ}$ au $12^{\circ}$; le segment céphalique est fort réduit et le postcéphalique est le plus long. La forme du corps est ainsi cylindroïde à extrémité antérieure atténuée conique, à extrémité postérieure arrondie; elle rappelle déjà celle de la larve tertiaire.

Le segment céphalique est terminal, avec une légère inclinaison ventrale ; il présente en avant deux renflements surbaissés portant

Annales de Parasitologie, T. I, No 4 . - Octobre 1923. 
à leur sommet deux points ocelliformes (fig. 2). En arrière et ventralement font saillie 3 paires de crochets buccaux : une paire de grands crochets fortement incurvés, à pointe aiguë, s'articulant en arrière avec l'appareil pharyngien ; en dehors de cette paire on oberve deux paires de crochets moins volumineux, dont l'externe est le plus petit ; il est aussi fort incurvé, tandis que l'incurvation des
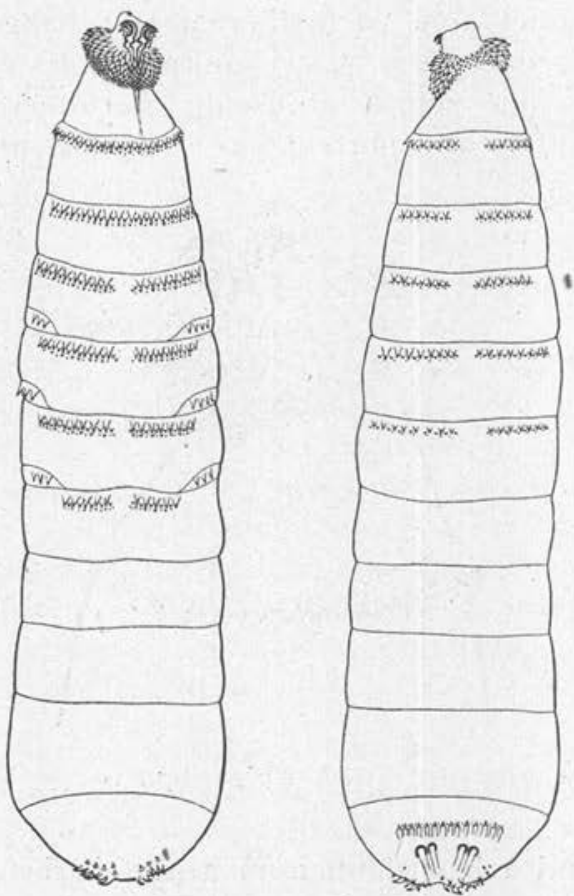

FIG. 1. - Cobboldia loxodontis, larve primaire : à gauche, vue ventrale; à droite, vue dorsale.

crochets moyens est moins accusée que celle des deux autres. Tous ces crochets ont leur pointe dirigée ventralement et en dehors ; sur une des larves nous avons observé les deux petits crochets d'un côté dirigés vers la face dorsale ; nous considérons cette disposition comme artificielle, résultant d'une déformation par écrasement de la larve.

Le segment postcéphalique est bien développé, conique, tronqué obliquement à sa partie antérieure, de telle sorte que sa face ventrale est moins développée que sa face dorsale. Son bord antérieur forme un bourrelet annulaire plus développé ventralement que 
dorsalement et muni d'une armature épineuse abondante, comprenant à la face ventrale 7 à 8 rangées d'épines hyalines alternes, dont les dimensions diminuent de la rangée antérieure à la postérieure; vers la ligne médiane dorsale, les épines deviennent plus petites et leurs rangées se réduisent à 4 ou 5 .

Le bord antérieur des anneaux 3 à 8 est armé à la face ventrale d'une ceinture épineuse composée de 4 rangées d'épines alternes, hyalines, à sommet aigu particulièrement réfringent; leur taille diminue de la première à la quatrième rangée, les épines de celle-ci étant réduites à une pointe minuscule ; certaines grandes épines de la rangée antérieure portent sur les côtés une petite pointe

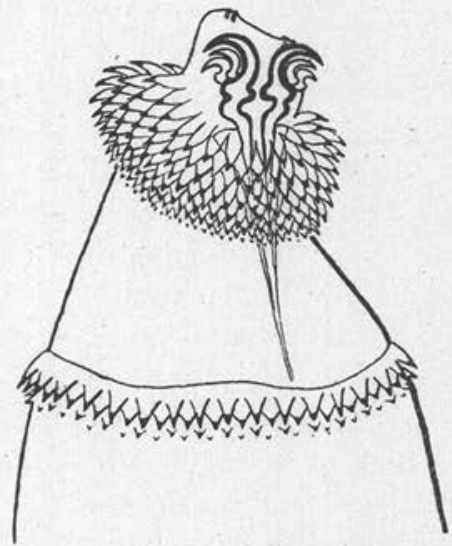

Fı́. 2. - Larve primaire : extrémité céphalique vue par la face ventrale.

latérale, qui tend à leur donner un aspect barbelé. Les ceintures des anneaux 3 et 4 sont entières, les suivantes sont interrompues sur la ligne médiane. A la face dorsale, les anneaux 3 à 7 sont munis d'une ceinture interrompue au milieu et formée par 3 rangées d'épines. Ces ceintures dorsales sont séparées sur les flancs des ceintures ventrales par un espace nu, sauf sur l'anneau 3. Les épines des ceintures dorsales sont moins fortes que celles des ceintures ventrales.

Entre les anneaux 5 à 8 , on observe de chaque côté trois petits champs intermédiaires fusiformes, armés de 4 à 5 épines disposées sur un rang.

L'anneau 8 est inerme à la face dorsale, ainsi que les anneaux 9 à 11 sur les deux faces.

L'anneau 12 est hémisphérique et porte les stigmates postérieurs 
qui sont pourvus de deux orifices s'ouvrant vers la face dorsale. Ces stigmates sont en rapport avec deux chambres feutrées volumineuses. En arrière et ventralement et en correspondance des stigmates on voit deux groupes d'épines saillantes disposées sans ordre particulier ; en avant et dorsalement, vers le milieu de la longueur du segment existe une double rangée transversale d'épines étroites, allongées, dont un certain nombre porte des pointes latérales, qui leur donnent un aspect barbelé. Ces épines sont disposées tantôt en antéversion, tantôt en rétroversion; toutes les autres épines du corps étant régulièrement en rétroversion.

Larve secondaire. - L'unique exemplaire de ce stade que nous avons observé mesure $9 \mathrm{~mm}$., 5 de long sur $3 \mathrm{~mm}$., 2 de largeur maximum. Le tégument est de coloration blanche. Le corps est de forme allongée, légèrement déprimé, à extrémité antérieure plus atténuée que la postérieure, toutes deux à contour arrondi. Sur les côtés existent des champs latéraux au nombre de deux sur les anneaux 3 à 11, les ventraux étant plus développés que les dorsaux correspondants. Un champ intermédiaire fusiforme se remarque en outre de chaque côté entre les anneaux 5 et 6,6 et 7,7 et 8 .

La longueur des anneaux augmente jusqu'au $6^{\circ}$, reste sensiblement invariable sur les anneaux 7 à 10 , augmente légèrement sur le $11^{\circ}$ qui est le plus long et se réduit sur le $12^{\circ}$. Leur largeur croît d'avant en arrière jusqu'au $7^{\circ}$ anneau pour diminuer régulièrement en arrière. Le développement des champs latéraux augmente de même jusqu'au $7^{\circ}$ anneau pour décroître au delà.

Le segment céphalique est petit, subterminal et ventral ; il présente pour le surplus la même conformation qu'au $3^{\circ}$ stade. L'anneau post-céphalique est fort développé, de forme conique et à axe incurvé ventralement; il est obliquement tronqué en avant et entoure l'anneau céphalique par son bord antérieur, dont la moitié dorsale occupe ainsi une situation apicale. Ce bord antérieur forme un bourrelet annulaire armé d'épines disposées en rangées multiples, plus nombreuses et à épines plus fortes ventralement que dorsalement. Vers le bord postérieur du même anneau, on voit de chaque côté le stigmate antérieur sous forme d'un nodule de coloration brunâtre. Observé au microscope sur la larve encore enfermée dans le tégument du stade précédent, ce stigmate se montre formé par une plaque ovoïde perforée par 7 ou 8 ostioles. Le segment terminal est semi-globuleux et montre la fente stigmatique limitée par deux lèvres : une ventrale épaisse, transversale, sillonnée sur la ligne médiane et munie de deux fortes papilles coniques ; 
sa face interne est parsemée d'épines; la lèvre dorsale à peine saillante décrit un demi-cercle et porte quatre papilles coniques volumineuses. Les plaques stigmatiques présentent une forme semi-lunaire, à bord externe arrondi, à bord interne rectiligne ; leur contour externe est marqué par un cadre brun de chitine ; elles sont largement séparées l'une de l'autre et portent chacune deux arcades rectilignes verticales. Au-dessus de ces plaques et au bord interne de la lèvre dorsale existe une armature épineuse formée de 3 à 4 rangées d'épines allongées, alternes, dont les plus internes sont les plus volumineuses.

L'armature des anneaux du corps est distribuée comme suit : à la face ventrale 3 rangées sur les anneaux 3 et 6 à 8 , et 4 rangées sur les anneaux 4 et 5 ; à la face dorsale 3 rangées sur les anneaux 3 à 5 et 2 rangées sur les anneaux 6 et 7 . Toutes les ceintures sont interrompues au milieu tant à la face ventrale qu'à la face dorsale, à l'exception des ceintures ventrales sur les anneaux 3 et 4 . Les ceintures ventrales se poursuivent sur les champs latéraux ventraux et sont séparées des dorsales par un espace nu au niveau des champs latéraux dorsaux. Les champs intermédiaires latéraux sont armés d'une rangée de fortes épines. Toutes les épines sont hyalines, à sommet aigu à peine teinté.

Larve tertiaire. - A l'état de complet développement, elle peut acquérir une taille de 20 à $28 \mathrm{~mm}$. et un maximum de largeur de $8 \mathrm{~mm}$. La coloration du tégument est blanche chez les exemplaires jeunes, brun clair chez les individus mûrs.

Le corps est de forme allongée, faiblement déprimé sur la face ventrale, plus longuement et plus fortement atténué à son extrémité antérieure, qui est conique à sommet mousse et incurvé ventralement, qu'à son extrémité postérieure qui est arrondie (fig. 3).

La longueur des anneaux augmente d'avant en arrière jusqu'au $8^{e}$ ou $9^{e}$ pour diminuer au delà ; leur largeur croît de même jusqu'au $8^{\circ}$ anneau et décroît en arrière. Sur les côtés existent deux champs ou bourrelets latéraux sur les anneaux 3 à 11, d'autant mieux délimités qu'ils occupent des anneaux plus postérieurs ; leur développement augmente du $3^{\circ}$ au $6^{\circ}$ ou $7^{\circ}$ et diminue progressivement en arrière. Les champs latéraux ventraux des anneaux 5 à 11 portent une papille sensorielle, obsolète sur les anneaux 5 à 8 , bien apparente sur les anneaux 9,10 et 11 ; le développement de cette papille augmente d'avant en arrière, sans toutefois jamais devenir saillante comme chez Rodhainomyia chrysidiformis. Entre les champs latéraux ventraux des anneaux 5 à 9 s'interposent 
de chaque côté quatre champs intermédiaires fusiformes, dont les $2^{\circ}$ et $3^{e}$ sont les plus développés.

Le segment céphalique est petit, subterminal, dirigé vers la face ventrale et de forme trapézoïdale. Il porte en avant deux renflements antennaires globuleux, largement séparés à leurs bases ; ils sont dirigés en dehors et munis à leur sommet de deux points ocelliformes. Plus en arrière et plus au milieu font saillie les deux crochets buccaux brun foncé, fortement incurvés, à pointe
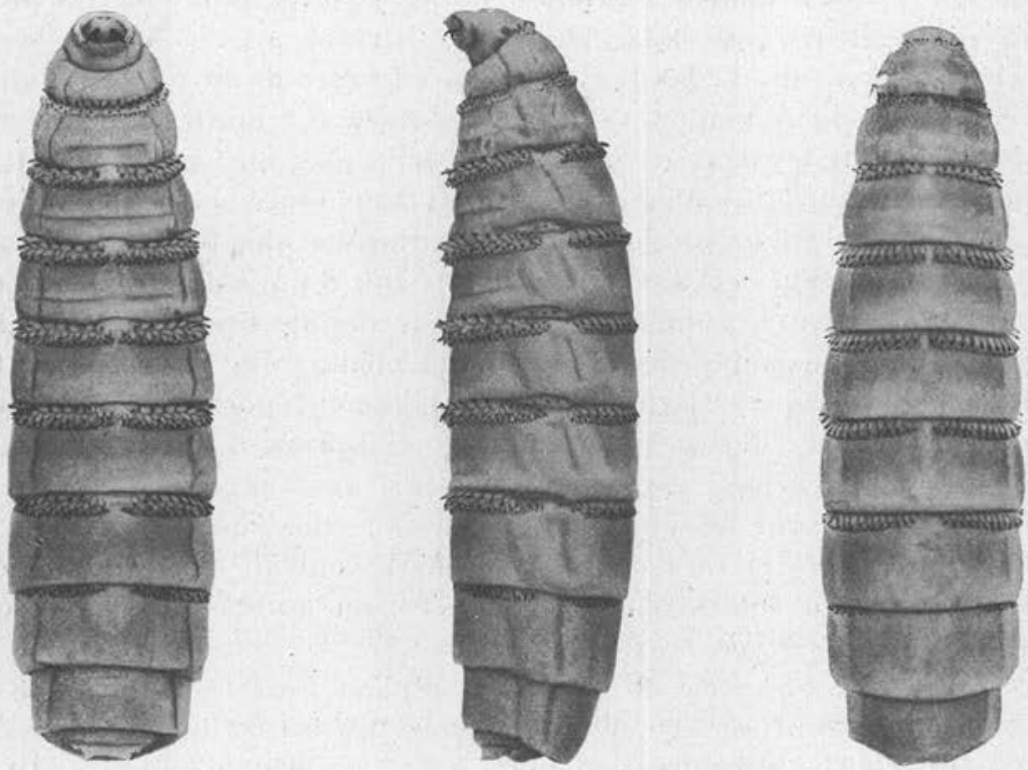

Fig. 3. - Cobboldia loxodontis, larve tertiaire :

à gauche, vue ventrale; au milieu, vue latérale ; à droite, vue dorsale.

aiguë dirigée en dehors et en arrière. Le tégument, en arrière des renflements antennaires et en dehors des crochets buccaux, est parsemé de minuscules épines à sommet dirigé en avant et en dehors.

L'anneau post-céphalique est conique, à axe incliné ventralement, à sommet tronqué obliquement. Son bord antérieur légèrement saillant entoure le segment céphalique qu’il déborde apicalement; il est armé d'une couronne d'épines disposées en rangées plus nombreuses ventralement que dorsalement. Vers le bord postérieur de l'anneau et sur les côtés se voient, au fond d'une dépression infundibuliforme du tégument, les stigmates antérieurs sous 
la forme d'un organe flabelliforme surmonté de 12 digitations disposées en fer à cheval.

L'anneau terminal est de forme subhémisphérique et présente deux lèvres transversales qui délimitent la fente stigmatique : la lèvre dorsale, la plus développée, porte quatre papilles coniques volumineuses, deux submédianes et deux latérales; la lèvre ventrale est parcourue par un sillon vertical qui la divise en deux moitiés portant chacune une papille submédiane. Les plaques stigmatiques sont ovalaires, à grand axe divergeant vers la face ventraie et entourées par un cadre chitineux, surtout accusé sur le bord externe ; chacune d'elles porte trois péritrèmes de forme rectiligne, divergeant légèrement vers la face dorsale et dont l'externe est plus petit que les deux internes. Les péritrèmes sont recouverts par une membrane cuticulaire ménageant une fente ouverte (Enderlein) sur toute leur longueur et soutenue dans la profondeur par des demi-arceaux chitineux qui alternent d'un côté à l'autre et s'unissent entre eux dans la profondeur par de fines travées obliques, dont l'ensemble décrit une ligne médiane en zigzag (1). Audessus des plaques stigmatiques, le tégument porte vers le bord libre de la lèvre dorsale une armature composée d'épines alternes, longues et barbelées, disposées sur plusieurs rangées et dont les plus internes sont les plus volumineuses; elles sont dirigées avec leur pointe vers la face dorsale. La lèvre ventrale porte à sa face interne de minuscules épines ordonnées, au nombre de 3 à 6 , en séries linéaires transversales.

L'armature épineuse du corps est répartie sur les anneaux 3 à 12 à la face ventrale et sur les anneaux 3 à $9-10$ à la face dorsale et comporte des ceintures composées de 4 rangées d'épines sur les anneaux ventraux 3,4 et 10 , de 5 rangées sur les anneaux 5 et 9 , de 6 rangées sur les anneaux 6 à 8 et de 2 à 3 rangées sur les an-

(1) Enderlein, qui a étudié la structure des stigmates du Cobboldia elephanlis, a signalé que les arceaux chitineux qui soutiennent la membrane cuticulaire ne sont pas lous égaux en développement : il a décrit des rudiments d'arceaux régulièrement interposés entre les arceaux complets. En 1916, nous avons étudié la structure de ces mêmes organes chez le Cobboldia loxodontis et avons reconnu que, chez cette espèce. tous les arceaux chitineux sont également développés, dispositif qui, pensions-nous, constituait un caractère différentiel des deux espèces de Cobboldia. Depuis il nous a été donné d'examiner des larves de Cobboldia elephantis, que nous devons à la gracieuse obligeance de M. Stanley Kemp, le savant directeur du Service zoologique de l'Inde. Nous sommes heureux de pouvoir lui renouveler ici l'expression de notre vive gratitude. Or à l'examen des plaques stigmatiques de l'espèce indienne, nous avons coustaté que la structure des péritrèmes ne diffère pas de celle de l'espèce africaine; c'est tout au plus si nous avons pu reconnaître deux ou trois arceaux rudimentaires qui sont probablement en rapport avec l'état de croissance non encore achevée des plaques stigmatiques de l'exemplaire examiné. Nous pensons done que l'exemplaire de Cobboldia elephantis qui a fait l'objet des études de Enderlein était un individu jeune encore en pleine croissance. 
neaux 11 et 12 ; à la face dorsale, les ceintures comprennent 4 à 5 rangées sur les anneaux 3 à 6,3 à 4 rangées sur les anneaux 7 et 8,3 rangées sur l'anneau 9 et $0-2$ rangées sur l'anneau 10.

Les ceintures ventrales sont entières sur les anneaux 3 et 4 , interrompues sur les anneaux suivants; sur les anneaux 5 à 7 cette interruption ne porte que sur les rangées antérieures d'épines; sur les suivants, elle s'étend à toutes les rangées et augmente d'étendue de l'anneau 8 à l'anneau 12 ; sur les derniers anneaux la ceinture se trouve ainsi réduite à deux groupes latéraux de petites épines qui, dans certains cas, peuvent faire défaut. Sur l'anneau 12, indépendamment des deux groupes latéraux, on observe parfois un groupe médian formé de 2 à 4 petites épines. A la face dorsale, les ceintures des anneaux 6 et 7 sont entières ; toutes les autres sont interrompues sur la ligne médiane; cette interruption est étroite sur les anneaux 3 à 5 et 8 , large sur l'anneau 9, davantage encore sur l'anneau 10, où l'on ne voit que deux petits groupes latéraux d'épines, qui peuvent, dans certains cas, ne comporter qu'une épine ou même manquer complètement.

Les ceintures ventrales et dorsales se poursuivent sur les champs latéraux des anneaux 3 à 10 ; elles sont toutefois séparées les unes des autres par un court espace nu au niveau des champs dorsaux sur les anneaux 3 et 4 , à la limite entre les champs ventraux et dorsaux sur les anneaux 5 à 10 et cette interruption augmente progressivement sur les anneaux postérieurs, de telle sorte que le champ dorsal 10 est toujours inerme et le champ dorsal 9 également ou presque, tandis que les champs ventraux 9 et 10 ne sont armés que sur leur moitié ventrale.

Les champs latéraux intermédiaires sont armés d'une rangée unique de fortes épines au nombre de 3 à 6 .

Le développement des ceintures augmente à la face ventrale jusque sur les anneaux 7 ou 8 , à la face dorsale jusque sur les anneaux 6 ou 7 et décroît en arrière.

Sur une même ceinture, la taille des épines diminue de la rangée antérieure à la postérieure ; en outre, sur les anneaux 5 à 10 , la taille des épines croît sur une même rangée de la ligne médiane vers le quart latéral pour décroître au delà, de telle sorte que les ceintures semblent formées de deux moitiés ou aires épineuses à contour postérieur convexe en arrière.

Cette disposition est surtout bien marquée à la face ventrale ; elle est moins prononcée à la face dorsale.

Toutes les épines sont à base de même coloration que le tégument et à sommet aigu légèrement teinté de brun. 
Si nous comparons les descriptions que nous venons de donner des trois stades larvaires de Cobboldia loxodontis, nous sommes frappés des analogies morphologiques que présentent ceux-ci ; dès le stade primaire, la larve accuse les caractères généraux de la conformation du stade tertiaire et le polymorphisme larvaire qui s'observe chez les Oestrides appartenant à d'autres sous-familles n'existe pas chez les Cobboldiinæ. Un autre caractère de groupe semble consister dans l'armature buccale du stade primaire, où l'on observe 3 paires de crochets buccaux ; Rodhain et Bequaert ont, en effet, signalé la même disposition chez la larve primaire de Rodhainomyia chrysidiformis, type d'un genre qui présente d'étroites affinités avec le genre Cobboldia.

Au cours des observations que nous avons pu faire récemment sur d'assez nombreuses larves de Cobboldia, nous avons réussi à résoudre une question se rattachant à ce genre de parasites. Dans un travail précédent, nous avions, en effet, cru avec Brauer pouvoir, sur la foi de la description qui en avait été donnée, distinguer des larves de Cobboldia loxodontis, la larve étudiée par R. Blanchard et qui avait été recueillie par le $\mathrm{D}^{\mathrm{r}} \mathrm{J}$. Kirk dans l'estomac d'un éléphant dans le bassin du Zambèze au cours de l'expédition de Livingstone, et nous avions proposé de la dénommer Cobboldia parumspinosa. Assez fréquemment nous avions rencontré des difficultés à déterminer exactement le nombre des rangées d'épines sur les ceintures des différents anneaux et à reconnaître la présence des champs intermédiaires latéraux et nous avions été pris de doute au sujet de l'exactitude de la description de R. Blanchard. Nous avons, en conséquence, exprimé le désir de revoir les matériaux originaux : grâce à l'aimable intervention de notre savant collègue, M. Baylis, du British Museum, nous avons obtenu des conservateurs du Museum du Royal College of Surgeons of England à Londres, Sir Arthur Keith et $D^{r}$ R.-H. Burne, la grande faveur de les recevoir en communication. Nous tenons à exprimer ici toute notre reconnaissance à ces distingués collègues pour leur grande obligeance. Il résulte de l'examen auquel nous avons soumis les deux larves de Kirk qu'elles ne diffèrent en rien de celles de Cobboldia loxodontis et que, par conséquent, Cobboldia parumspinosa tombe en synonymie. 